\title{
GENERALIZED NOISELESS QUANTUM CODES UTILIZING QUANTUM ENVELOPING ALGEBRAS
}

\author{
MICHO ĐURĐEVICH*, HANNA E. MAKARUK**, ROBERT OWCZAREK**
}

\begin{abstract}
*Instituto de Matematicas, UNAM, Area de la Investigacion Cientifica, Circuito Exterior, Ciudad Universitaria, Mexico DF, CP 04510, Mexico

**MS E517, E-ET, Los Alamos National Laboratory, Los Alamos, New Mexico 87545
\end{abstract}

Short Title: Generalized Noiseless Quantum Codes,

PACS No: 03.67.Lx, 02.20.Os, 02.20.Sv 
AbStract. A generalization of the results of Rasetti and Zanardi concerning avoiding errors in quantum computers by using states preserved by evolution is presented. The concept of the dynamical symmetry is generalized from the level of classical Lie algebras and groups, to the level of a dynamical symmetry based on quantum Lie algebras and quantum groups (in the sense of Woronowicz). An intrinsic dependence of the concept of dynamical symmetry on the differential calculus (which holds also in the classical case) is stressed. A natural connection between quantum states invariant under a quantum group action, and quantum states preserved by the dynamical evolution is discussed. 


\section{INTRODUCTION}

Quantum computation is a new and quickly developing area of science. Its power comes from using quantum parallelism of computations. This new paradigm for computation was envisioned by Feynman [1]. For many years quantum computation looked as an unrealistic dream. The reason is unavoidable decoherence due to the interaction of quantum devices with a classical environment, which destroys quantum coherent states. Then the advantage of parallelism is lost, and this makes quantum computation impossible. This situation changed radically when the quantum error correcting codes were invented [2, 3, 4]. This fact plus remarkable progress in experimental manipulation with individual qubits make the dream coming true.

In this paper we study a special implementation of so-called noiseless quantum codes, also known as error avoiding quantum codes [5]. Such codes were proposed in [6, 7] as an alternative or, more likely, supplement to the error correcting quantum codes. In [6, 7] error avoiding quantum codes were built using group theoretic methods. The idea is that among quantum states of the system there exist distinguished ones which, despite interaction with the environment, do not underlie decoherence. Important assumption was that qubits of the quantum register interact with a coherent environment. This assumption, besides the assumption on dynamical symmetry of the system, turned out to be essential for the introduction of the states protected against decoherence. Namely, it is possible to introduce collective variables describing the qubits composing the register. The singlet state of the qubits turned out to be protected against corruption.

An attempt to describe a more general situation was made in [8]. There the semigroup technique was used to describe general evolution of the system interacting with environment. In comparison to [6, 7], the generalization consisted in consideration of various degrees of coherence of the environment on the distances of the order of the length of the register. Besides full coherence, lack of any coherence and partial coherence were considered, too. Basic results on error protected states

are similar to the ones obtained in [6, 7]. Additional noises were considered, and it was shown that their influence on the error protected states is negligible up to the first order of a small parameter characterizing the noise. Performing quantum computations with the error protected states was shown to be realistic.

In the paper [5] general criteria for error avoiding quantum codes were formulated. First, the existing codes were divided into three groups: error correcting codes, error avoiding codes and error preventing codes. Error correcting codes detect and correct errors. Error preventing codes only detect errors, without correcting them. After the classification was made the general theory of error avoiding codes was formulated, in the manner following the paper [9], where the general theory of error correcting quantum codes was presented. It was found that the error avoiding codes are derived from the subspaces of the Hilbert space that are common eigenspaces of the operators $A_{a}$ describing the evolution of the system. If $\rho_{i}$ and $\rho_{f}$ are the initial and the final density matrices of the system respectively, then, under assumption that initially the system is not entagled with the environment, $\rho_{f}=\sum_{a} A_{a} \rho_{i} A_{a}^{+}$, where $\sum_{a} A_{a}^{+} A_{a}=I$.

¿From the paper [9] the conditions for the error correcting codes are known. Namely, if the vectors $\mid i>$ form an orthonormal basis of the code, the condition

$$
<i\left|A_{a}^{+} A_{b}\right| j>=\gamma_{a b} \delta_{i j}
$$


should be satisfied, where $\gamma_{a b}$ is a Hermitian matrix. All known error correcting codes have $\gamma_{a b}$ nondegenerate. The matrix is then expressible, after some unitary redefinition of $A_{a}$, as a diagonal matrix with positive entries.

As shown in [5] error avoiding codes also satisfy (1.1) but are maximally degenerate (in the diagonal form only one diagonal element is different from zero). Moreover the matrix $\gamma_{a b}$ is expressible as $\gamma_{a b}=\gamma_{a}^{*} \gamma_{b}$, where $\gamma_{a}$ are eigenvalues of the respective operators $A_{a}$ for the states from the code. This general approach does not use the group theoretic language, so that we do not know if there exist error avoiding codes of different origin than the group theoretic (or quantum group theoretic) one. It shows however usefulness of the error avoiding codes for quantum computing (especially when used simultaneously with the error correcting codes).

The aim of this paper is to study error avoiding codes in a more general framework than the group-theoretic one. More precisely, we shall discuss the problematics of error avoiding codes in the framework of quantum groups.

Our motivation is that the quantum group framework enables introduction of a more general dynamical symmetry of the system, comparing to the standard group theoretic one. By construction, it covers also the dynamical symmetry connected with classical groups, since the classical groups are all special cases of quantum groups. We hope to take this way into account certain fluctuations from the exact group theoretic dynamical symmetries required by the standard noiseless codes.

In [6, 7] there was expressed a hope that deviations from the proposed ideal situation should not destroy too much of the quantum coherence and consequently the error protected states should become 'almost error protected' under these conditions. Our considerations show there exist particular perturbations for which the error protected states 'remain' exactly protected despite these perturbations.

The plan of the paper is as follows. In the second section we define and briefly discuss the notion of dynamical symmetry connected with quantum groups. In the third section we formulate and prove main theorems concerning error protected states. In the fourth section we present simple examples to illustrate the general results. We conclude the paper with the discussion of possible extensions of this work. In the Appendix we give a very brief review of the basic material on quantum groups and their representations.

\section{Dynamical Symmetry Coming From Quantum Groups}

Symmetry proved to be one of the basic notions in physics. Dynamical symmetry of a physical system is defined in terms of its Hamiltonian, which should be expressible as a linear combination of operators generating a representation of the appropriate Lie algebra. There is a large class of systems possessing such a property. Dynamical symmetry of a system should not necessary be visible at a first sight. Nevertheless, searching for such a symmetry is highly rewarding, since one can apply to the systems with a dynamical symmetry powerful methods developed on the ground of the theory of Lie algebras and their representations, like the method of coherent states [10]. Dynamical symmetry proved to be also important in searching for physical systems possessing very specific quantum states-which can not be corrupted despite their interaction with the environment [6, 7]. Such systems provide noiseless quantum codes that are of potential great interest for constructing quantum computers. Noiseless quantum codes can be either alternative to error correcting codes, which are elaborate methods of coding information, 
recognizing errors and correcting them [2, 3, 4, or a valuable supplement to such codes.

It turns out that analogous codes exist for systems with dynamical symmetry based on quantum groups instead of Lie groups. The goal of this section is to define the notion of dynamical symmetry associated to quantum groups. In the next section we shall apply our new concept of dynamical symmetry to prove our main theorems concerning error protected states. Then, we shall study some systems providing noiseless quantum codes.

Basic mathematical concepts and tools that will be used in the paper are briefly presented in the Appendix.

A generalization of the concept of dynamical symmetry can be defined only when there are well established notions of a Lie algebra, and the corresponding universal enveloping algebra, associated to a given quantum group $G$. In the theory of quantum groups, all these notions essentially depend on an appropriately chosen differential calculus over $G$.

The quantum group $G$ will be represented by a non-commutative $C^{*}$-algebra $A$, playing the role of the algebra of 'continuous functions' defined on the quantum space $G$, together with a coproduct map $\phi: A \rightarrow A \otimes A$ (corresponding to the standard product in the case of classical groups). Effectively, all caclulations will be performed within an everywhere dense ${ }^{*}$-subalgebra $\mathcal{A} \subseteq A$, playing the role of polynomial functions over $G$. Actually, $\mathcal{A}$ is a Hopf *-algebra in a natural way.

Suppose that on $G$ is defined a *-covariant, left-covariant first-order differential calculus $\Gamma$. Let $L$ be the associated quantum Lie algebra. If the calculus $\Gamma$ is in addition right-covariant, we can introduce the universal enveloping algebra $U(L)$. Every representation $v: V \rightarrow V \otimes \mathcal{A}$ of $G$ in a finite-dimensional vector space $V$ naturally induces (as in the classical theory) a representation $\delta=\delta_{v}$ of $L$ and $U(L)$ in $V$.

Definitions of all these objects are sketched in the Appendix.

We consider an open quantum system, represented by a Hilbert state space $V=H_{S}$. The system interacts with its environment (bath) which is described by a Hilbert space $H_{B}$. Here it is assumed for simplicity that all Hilbert state spaces are finite dimensional - however, everything could be incorporated into the infinite-dimensional case.

Definition 2.1. We say that a system has quantum dynamical symmetry described by the quantum group $G$ and its quantum Lie algebra $L$ if the following conditions are satisfied:

(i) The evolution of the system is governed by the Hamiltonian

$$
\mathcal{H} \in \operatorname{End}\left(H_{S} \otimes H_{B}\right) \simeq \operatorname{End}\left(H_{S}\right) \otimes \operatorname{End}\left(H_{B}\right) .
$$

(ii) The Hamiltonian is a hermitian operator $\mathcal{H}^{*}=\mathcal{H}$.

(iii) The Hamiltonian is of the form:

$$
\mathcal{H}=P_{1}\left(l_{1}, \ldots, l_{n}\right) \otimes T_{1}+\ldots+P_{N}\left(l_{1}, \ldots, l_{n}\right) \otimes T_{N}
$$

where $P_{1}, \ldots, P_{N}$ are polynomial expressions of infinitesimal generators $l_{i}=\delta\left(e_{i}\right)$ and $\left\{e_{i}\right\}$ is a basis in $L$. Finally $T_{1}, \ldots, T_{N}$ are hermitian operators

$$
T_{\alpha}: H_{B} \rightarrow H_{B}
$$


Such systems with quantum dynamical symmetry can be explored by generalized methods known from the theory of systems with classical dynamical symmetry, for example by the method of quantum coherent states [11]. Let us observe that the terms in (2.1) can be reorganized in such a way, that the Hamiltonian takes a more familiar form:

$$
\mathcal{H}=\mathcal{H}_{S} \otimes \mathrm{id}+\mathrm{id} \otimes \mathcal{H}_{B}+\mathcal{H}_{I}
$$

where $\mathcal{H}_{S}$ is the system's Hamiltonian, $\mathcal{H}_{B}$ is the Hamiltonian of the environment and $\mathcal{H}_{I}$ is the 'interaction hamiltonian' uniquely defined as the part of $\mathcal{H}$ traceless in both tensor factors.

\section{Error Protected States \& Noiseless Quantum Codes}

In this section we present our main theorems on error protected states, and on noiseless quantum codes. We assume that we deal with a (open) quantum system with dynamical symmetry of a quantum group, and all other features as described in the previous section. The vectors that are $v$-invariant, where $v$ is a representation of the quantum group $G$, are of vital importance for our further discussion. Let us give their definition now. Let $v: V \rightarrow V \otimes \mathcal{A}$ be an arbitrary representation of $G$ in a finite-dimensional vector space $V$, and let $\delta=\delta_{v}: L \rightarrow \operatorname{End}(V)$ be the associated representation of $L$. To further simplify the considerations, we shall consider the case when the quantum group is 'connected' in the sense that only scalar elements of $\mathcal{A}$ are annihilated by the differential $d: \mathcal{A} \rightarrow \Gamma$.

Then the following equivalence holds for every vector $\psi \in V$

$$
v(\psi)=\psi \otimes 1 \quad \Leftrightarrow \quad \delta(x) \psi=0, \quad \forall x \in L
$$

Let us assume that the calculus $\Gamma$ is in addition bicovariant. This enables us to introduce the quantum universal enveloping algebra $U(L)$, and to discuss the representations of $U(L)$ associated with the representations of the quantum group $G$. Let us also introduce the map $\chi: U(L) \rightarrow \mathbb{C}$, with the properties $\chi(L)=0$, $\chi(1)=1$, extended then to the whole $U(L)$ by multiplicativity. The representation $\delta$ uniquely (as in the standard theory) extends from $L$ to $U(L)$. The above two conditions are further equivalent to

$$
\delta(q) \psi=\chi(q) \psi, \quad \forall q \in U(L) .
$$

The proof of these equivalences is quite straightforward, but it needs some additional definitions and constructions, which we would rather skip in this paper as they are going too far in the formalism. Vectors satisfying any of the above conditions are called v-invariant. The $v$-invariant vectors are very important for the study of quantum registers (which are open systems with a quantum dynamical symmetry). Such vectors give us examples of the error protected states.

Our main theorem reads:

Theorem 3.1. Unitary evolution described by the Hamiltonian $\mathcal{H}$ possessing a quantum dynamical symmetry given by $(G, L)$ preserves the $v$-invariant vectors and associated states of the system, even when the other states of the system are corrupted due to decoherence. 
Proof. Let us take as an initial vector $\psi \otimes \zeta \in H_{S} \otimes H_{B}$, where $\psi$ is $v$-preserved in the sense defined above. Then the unitary evolution defined by

$$
U(t)=\exp \left(-\frac{i}{\hbar} \mathcal{H} t\right)
$$

with $\mathcal{H}$ of the form (2.2) gives

$$
\exp \left(-\frac{i}{\hbar} \mathcal{H} t\right)(\psi \otimes \zeta)=\psi \otimes \exp \left(-\frac{i}{\hbar} \mathcal{H}_{\text {eff }} t\right) \zeta
$$

where

$$
\mathcal{H}_{\text {eff }}=\chi\left(P_{1}\right) T_{1}+\ldots+\chi\left(P_{N}\right) T_{N}
$$

This proves the statement.

Interesting property of $\mathcal{H}_{\text {eff }}$ is that the coefficients $\chi\left(P_{i}\right)$ should somehow reflect the structure given by $G$ and its Lie algebra $L$.

Now we can easily prove generalization of the theorems 1 and 2 given in the paper [6]. We follow the notation from [6]. Let $\rho_{S} \in \operatorname{End}\left(H_{S}\right)$ and $\rho_{B} \in \operatorname{End}\left(H_{B}\right)$ be the (mixed quantum) states of the system and the environment respectively. If the overall system is initially in the state $\rho(0)=\rho_{S} \otimes \rho_{B}$, then $\rho(t)=U(t) \rho(0) U(t)^{+}$, so that the evolution is unitary. The induced evolution on $H_{S}$ is given exactly like in [6] by $L_{t}: \rho_{S} \rightarrow \operatorname{tr}_{B} \rho(t)$, where $\operatorname{tr}_{B}$ is the trace over $H_{B}$. Then the following theorem is fulfilled:

Theorem 3.2. Let $\mathcal{M}_{N}$ be the manifold of states built over the space of vectors invariant under the representation $v$, and $\rho_{S} \in \mathcal{M}_{N}$. Then for any initial bath state $\rho_{B}$ the induced evolution on $H_{S}$ is trivial,

$$
L_{t}\left[\rho_{S}(t)\right]=\rho_{S}, \quad \forall t>0 .
$$

Proof. Theorem 3.1 allows us to reduce the proof of 3.2 to the proof of the first theorem of [6].

The invariant vectors are generalizations of the singlet states pointed out in [6] as the states of the quantum register which are not corrupted by interaction with the environment.

We should stress that the Hamiltonian of the system + environment should not necessary contain terms with trivial representation in the space of the system and in the space of the environment, so that it can be even of the pure interaction form.

Before we present simple examples illustrating the general theory and explicitly showing the 'error protected' states, let us discuss interesting question of the structure of the Hilbert space of the quantum computer registers, and discuss physical implications. The register usually consists of a number of copies of the same quantum system, often having two possible states for example spin 'up' and spin 'down' (a qubit).

Dynamical symmetry is defined in the Hilbert space that originates from the Hilbert spaces for individual qubits being described as carrier spaces of unitary representations

$$
v_{i}: V_{i} \rightarrow V_{i} \otimes \mathcal{A} \quad i=1, \ldots, n
$$

of our quantum group $G$.

The register Hilbert space is the tensor product of the representation spaces,

$$
V=V_{1} \otimes V_{2} \otimes \ldots \otimes V_{n}
$$


in which $G$ acts by the direct product

$$
v=v_{1} \times v_{2} \times \ldots \times v_{n}
$$

of representations $v_{i}$. Each of the representations $v_{i}$ induces a representation $\delta_{i}$ of the corresponding quantum universal enveloping algebra. The representation $v$ induces the representation $\delta$ of the quantum universal enveloping algebra, and one can easily prove the following relation:

$$
\delta(x)\left(\phi_{1} \otimes \ldots \otimes \phi_{n}\right)=\sum_{k=1}^{n} \sum_{\alpha \in I[k]} \phi_{1} \otimes \ldots \otimes \delta_{k}\left(x^{\alpha}\right) \phi_{k} \otimes \eta_{k+1}^{\alpha} \otimes \ldots \otimes \eta_{n}^{\alpha}
$$

where

$$
\tau_{n-k}\left(\left\{\phi_{k+1} \otimes \ldots \phi_{n}\right\} \otimes x\right)=\sum_{\alpha \in I[k]} x^{\alpha} \otimes\left\{\eta_{k+1}^{\alpha} \otimes \ldots \otimes \eta_{n}^{\alpha}\right\}
$$

and $\tau_{n-k}: V_{k+1} \otimes \ldots \otimes V_{n} \otimes L \rightarrow L \otimes V_{k+1} \otimes \ldots \otimes V_{n}$ are the appropriate 'flip-over' operators naturally associated to the differential calculus.

The formula (3.1) differs from the corresponding formula for the classical case of addition angular momenta in quantum mechanics ( $\tau$ is in the classical case just the standard transposition). It is easy to see that qubits in the register are not treated on the same footing. It could be associated to some effects due to, not taken into account in [6], linear extension of the register, or to fluctuations of the fields due to nonideal structure of boundaries of the register and their influence. Anyway, it is possible to realize a system with weaker symmetry than the one presented in [6] but still possessing error protected states. It is known that similar deviations from exact dynamical symmetry of Lie groups lead to better mass (or energy) formulas in both nuclear/particle physics, and molecular physics [14, 15]. Therefore, one can look also among such systems for possible candidates for registers of quantum computers.

In [6] a physically plausible conjecture was expressed, that small deviations from ideal properties assumed of the system should lead to small errors in the errorprotected states. Actually, we have shown that there exist systems with special kind of deviation from the assumed symmetry, which nevertheless still have error protected states.

\section{EXAMPLES}

Let us switch now to some simple examples that would highlight our general ideas. The first example of a quantum group presented systematically in the literature was a quantum version of the standard $S U(2)$ group 16 , where the theory of representations was developed together with various geometrical aspects and a construction of a natural three-dimensional left-covariant differential calculus. This calculus is not bicovariant, and the minimal dimension for a bicovariant calculus over the quantum $S U(2)$ is 4 (this four-dimensional calculus 12 is analyzed in detail in [17]). Generalization of the results concerning this particular quantum group leads to the general theory of compact matrix quantum groups [18, 12], the definition of quantum spheres [19] and their geometry [20, deep generalization of the Tannaka-Krein duality [13], and also the theory of quantum principal bundles together with the corresponding gauge theory on quantum spaces, first formulated in [21] and then developed systematically in [22, 23] (see also [24, 25, 26]). Also in the $C^{*}$-algebraic framework the quantum homogeneous bundles were defined and 
the example of such a bundle with quantum spheres as fibers was given [27. Different approaches to quantum groups were developed in 28, 29, where quantum groups are treated from the point of view of deformations of universal enveloping algebras, Yang-Baxter equations and completely solvable systems.

We shall use the quantum version of $S U(2)$ in our examples. This is relatively simple from computational viewpoint, but highly non-trivial and very suggestive for the aims of this paper. First, we remind some basic facts about this group, which is denoted by $S_{\mu} U(2)$. Here the deformation parameter $\mu$ takes the values $\mu \in[-1,1] \backslash\{0\}$, and $\mu=1$ corresponds to the classical $S U(2)$ group.

In our further considerations important role will play the fact that irreducible unitary representations of $S_{\mu} U(2)$ are classified by the half-integers, like the representations of $S U(2)$. The fundamental representation corresponds, as in the classical case, to spin $j=\frac{1}{2}$ (see Appendix for more details). The Clebsch-Gordan decompositions of tensor products of the representations of the $S_{\mu} U(2)$ into irreducible representations look similar (concerning the multiplicities of the appearance of irreducible components in the products of representations) as in the classical case:

$$
\overbrace{u \times \cdots \times u}^{k}=\bigoplus_{j \in J} n_{j, k} u_{j}
$$

with the numbers $n_{j, k}$ the same as in the classical case. In particular, the decomposition of the second tensor power of the fundamental representation is $u_{1 / 2}^{2}=u_{0} \oplus u_{1}$, where $u_{0}$ and $u_{1}$ are the 1 -dimensional and the 3 -dimensional irreducible representations, respectively. One can describe these representations more explicitly after introducing the orthonormal basis in the representation space $V=\mathbb{C}^{2}$ of $u_{1 / 2}$, which will be denoted $|+\rangle,|-\rangle$ for the purpose of being easily recognizable by physicists. The tensor square $u_{1 / 2}^{2}$ is realized in $V \otimes V \simeq \mathbb{C}^{4}$, and the orthonormal basis in this space is $|+\rangle \otimes|+\rangle,|+\rangle \otimes|-\rangle,|-\rangle \otimes|+\rangle,|-\rangle \otimes|-\rangle$. It is an easy exercise to find that the invariant subspaces of $u_{1 / 2}^{2}$ are spanned by:

$$
\frac{1}{\sqrt{1+\mu^{2}}}(|+\rangle \otimes|-\rangle-\mu|-\rangle \otimes|+\rangle)
$$

and

$$
\begin{gathered}
|+\rangle \otimes|+\rangle \\
\frac{\mu}{\sqrt{1+\mu^{2}}}\left(|+\rangle \otimes|-\rangle+\frac{1}{\mu}|-\rangle \otimes|+\rangle\right) \\
|-\rangle \otimes|-\rangle
\end{gathered}
$$

The formula (4.1) generalizes the standard singlet, and the formula (4.2) generalizes the standard triplet. In analogy to the classical case the even tensor powers of the fundamental representation decompose into irreducible representations in such a way that the one-dimensional representation appears a number of times, and the number is identical as in the classical case. These singlets will be preserved by the dynamics.

4.1. First Example. In the first example we treat a system which is very similar to the one considered in [6]. Namely, as a model of the environment (bath) we 
consider a system of harmonic oscillators, described by the Hamiltonian

$$
\mathcal{H}_{B}=\sum_{k} \omega_{k} b_{k}^{\dagger} b_{k}
$$

acting in the Hilbert space $H_{B}$. The register consists in this simplest case of two qubits. In contrast to the case considered by Zanardi and Rasetti [6], the system consisting of the register and the bath has the dynamical symmetry not of the classical but of the quantum $S U(2)$ group. As already mentioned, in the quantum group context it is necessary to chose the differential calculus, prior to establishing the notion of the dynamical symmetry. The closest to the classical case seems to be introduction of the $3 D$ left-covariant calculus [16]. In other words, the quantum Lie algebra $L$ is 3 -dimensional. Let us denote by $K_{i}$ the operators representing the basis vectors $l_{i}$, in an arbitrary representation of $L$ (here $i \in\{1,2,3\}$ ). The following recurrent formulas enable us to compute explicitly the operators $K_{i}$, in the arbitrary tensor product of elementary 2-dimensional representations (qubits):

$$
\begin{aligned}
& K_{3}(\psi \otimes|+\rangle)=\frac{1}{2} \psi \otimes|+\rangle+\frac{1}{\mu^{2}} K_{3}(\psi) \otimes|+\rangle \\
& K_{3}(\psi \otimes|-\rangle)=\mu^{2} K_{3}(\psi) \otimes|-\rangle-\frac{1}{2} \psi \otimes|-\rangle \\
& K_{j}(\psi \otimes|+\rangle)=\frac{1}{2} \psi \otimes|+\rangle+\frac{1}{\mu} K_{j}(\psi) \otimes|+\rangle \\
& K_{j}(\psi \otimes|-\rangle)=\mu K_{j}(\psi) \otimes|-\rangle-\frac{1}{2} \psi \otimes|-\rangle,
\end{aligned}
$$

where $j \in\{1,2\}$.

In such a case the bath-register interaction Hamiltonian which is the quantum group analog of the Hamiltonian used in [6] reads:

$$
\mathcal{H}_{I}=K_{+} \otimes T+K_{-} \otimes T^{\dagger}+K_{3} \otimes T^{\prime},
$$

where $K_{ \pm}=K_{1} \pm i K_{2}$, and $T, T^{\prime}$ are operators acting in the bath Hilbert statespace. Relating to the corresponding formulas in [6], the operators $T$ and $T^{\prime}$ are obtained as the appropriate linear combinations of the creation and annihilation operators describing relevant elementary excitations of the bath. The operators $K_{j}$ are acting in the 4-dimensional 2-qubit space. In other words, it is formally of the same shape as in [6], however the 'spin' operators are different as explained above. The singlet state of the register is error-protected in the sense discussed above.

4.2. Second Example. In the second example the only difference with the first example is the register consists now of any even number of qubits, instead of just two. The spin operators $K_{j}$ are referring to the total register system, and are calculated by applying the above listed inductive rules (4.6).

It is important to stress that the number of singlet states is just the same as in the classical $S U(2)$ case. This is a consequence of the mentioned similarity between the representation theories for quantum and classical $S U(2)$ groups. The dimension of the singlet state space depends on the number of qubits in the way described in [6]. All these states are clearly protected from corruption due to decoherence.

4.3. Remark. The group $S U(2)$ appears as a dynamical symmetry group mainly in the context of the dynamics of spin systems. It appears less frequently in the context of dynamics of different systems, like bosonic particles. Therefore, the examples usually begin with the fundamental representation $u_{1 / 2}$ of $S U(2)$, as the elementary building blocks of the Hilbert space of the system. However, such a fundamental block could be any of $u_{j}$ with $j$ half-integer. Since for example $u_{j} \times u_{j}$ 
contains the singlet $u_{0}$ in its splitting into irreducible representations. this could be a starting point for building error protected states with the help of bosonic particles. Similar considerations are true also for the quantum group $S_{\mu} U(2)$. It seems, though, that a physical realization of such systems is more complicated and creates more technical problems.

Let us stress that in the examples we considered following the paper [6], all qubits are coupled to the same, coherent, environment. As was stressed in [30] coupling to the same environment of the qubits gives more possibility to get error protected states than coupling to independent environments. However, our methods seem to be general enough to deal with the cases of coupling with independent environments as well, till the system has a dynamical symmetry of the type introduced in this paper.

\section{Conclusions}

In this paper we introduced the general notion of dynamical symmetry associated with quantum groups and Lie algebras. Then we applied this notion to construct error protected states for open systems with such symmetry. The states can be useful for quantum computation. They are close analogs of their standard group theoretical counterparts. As a result, the error protected states obtained in strictly group theoretical dynamical symmetry context have counterparts preserved when the symmetry is slightly deviated towards the quantum group theoretic one. Recently various authors [31, 32] [33] [34] [35] introduced a technique of quantum computation which dynamically eliminates errors, by a quantum counterpart of the classical so called 'bang-bang' technique. Zanardi [36] has shown that the technique called 'symmetrizing' can be interpreted group theoretically as control of the systems forcing the systems with dynamical symmetry of a Lie group to be in states which are error protected. This very interesting observation should increase interest in error avoiding quantum codes. Since mathematically the technique seems to rely on invariant measures on the groups, it is applicable not only to the systems with the dynamical symmetry of finite groups discussed in the paper, but also to the systems with dynamical symmetry of the compact (even locally compact) Lie groups, which all possess Haar measure necessary for such construction. One should observe that the same is also true for compact (or locally compact) quantum groups, since these objects possess the Haar measure, too. It seems the generalization of the results by Zanardi to the quantum group case is straightforward, but its physical interpretations are less clear. Work on this issue is in progress.

\section{ACKNOWLEDGEMENTS}

M. Đurđevich likes to thank Theoretical Division of Los Alamos National Laboratory for the hospitality, H.E. Makaruk and R. Owczarek like to acknowledge the hospitality of the Math Institute of National Autonomous University of Mexico. Realization of this research was partially supported by Investigation Project in106879 of DGAPA/UNAM

\section{Appendix A. Quantum Spaces and Quantum Groups}

Classical theorem by Gelfand and Naimark states that compact topological spaces are in a natural correspondence with commutative unital $C^{*}$-algebras. These 
$C^{*}$-algebras consist of continuous complex-valued functions on the corresponding spaces.

Let $X$ be a compact topological space and $C(X)$ be the associated algebra of continuous complex-valued functions on $X$. The linear structure on $C(X)$ is given by the obvious conditions: $(f+g)(x)=f(x)+g(x)$, and $(\alpha f)(x)=\alpha f(x)$. The product in the algebra is $(f \cdot g)(x)=f(x) g(x)$ and the *-involution is given by $f^{*}(x)=\overline{f(x)}$.

There is a natural norm in $C(X)$ given by

$$
\|f\|=\sup \{|f(x)|: x \in X\} .
$$

In such a way is introduced a structure of (commutative) $C^{*}$-algebra in $C(X)$. Conversely, every commutative unital $C^{*}$-algebra is of this form-according to classical Gelfand-Naimark theory. Actually, the Gelfand-Naimark theory can be generalized to the level of locally-compact spaces, giving us a correspondence between arbitrary commutative (not necessarily unital) $C^{*}$-algebras and locally compact topological spaces. This correspondence is functorial, in the sense of category theory.

These facts lead to a generalized concept of space, which is understood as 'the underlying space' of a general $C^{*}$-algebra, about which we no longer assume it should be commutative. Generalized spaces of this type are called quantum spaces. The reason for the adjective 'quantum' follows from the observation that as in the classical quantization scheme a commutative algebra of functions is substituted by a noncommutative algebra of operators acting in a Hilbert space. The latter is indeed the case since all $C^{*}$-algebras can be realized as algebras of operators acting in some Hilbert spaces.

Interesting algebra and geometry appears when the classical topological spaces are equipped with an additional structure: differential-geometric, metric, Lie group, and so on. A very important class of quantum spaces constitute the quantum groups, which are understandable as quantum spaces equipped with a group structure.

Let us explain now, very briefly, what is exactly a compact quantum group. Let us start from a classical compact topological group $G$. This means that $G$ is a compact topological space equiped with a group structure, such that the product map $\circ: G \times G \rightarrow G$ is continuous (it can be shown that in the compact case continuity of the product implies continuity of the inverse map). At the dual level, the product map is represented by a ${ }^{*}$-homomorphism $\phi: A \rightarrow A \otimes A$, where $A=C(G)$.

More precisely, we first naturally identify

$$
\overbrace{A \otimes \cdots \otimes A}^{k}=C(\overbrace{G \times \cdots \times G}^{k}) \quad k \geq 2
$$

and define

$$
\phi(f)\left(g_{1}, g_{2}\right)=f\left(g_{1} g_{2}\right), \quad f \in A \quad g_{1}, g_{2} \in G .
$$

The associativity property of the product is equivalent to the coassociativity property

$$
(\phi \otimes \mathrm{id}) \phi=(\mathrm{id} \otimes \phi) \phi .
$$

It can be shown that the remaining two group axioms (the existence of the neutral element and the existence of the inverse elements) are equivalent to a single 
assumption that the elements of the form $a \phi(b)$ as well as of the form $\phi(b) a$, where $a, b \in A$, span two everywhere dense linear subspaces of $A \otimes A$.

Generalizing this to the quantum level, we define a group structure on a quantum space $G$ as a *-homomorhism $\phi: A \rightarrow A \otimes A$ such that the diagram

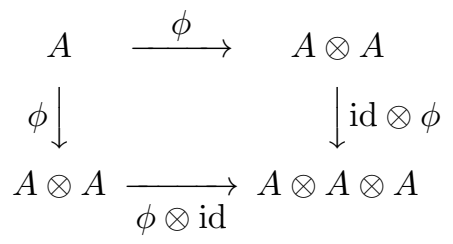

is commutative, and such that

$$
\begin{aligned}
& A \otimes A=\overline{\left\{\sum a \phi(b) \mid a, b \in A\right\}} \\
& A \otimes A=\overline{\left\{\sum \phi(b) a \mid a, b \in A\right\} .}
\end{aligned}
$$

where the bar means appropriate closure.

As a very important special case of this structure, let us mention matrix groups. These structures are given by triplets $(A, \phi, u)$ consisting of a $C^{*}$-algebra $A$, a *homomorphism $\phi: A \rightarrow A \otimes A$ and a matrix $u \in M_{n}(A)$ (all $n \times n$-matrices with coefficients from $A$ ) which is (together with the conjugate matrix $\bar{u}$ ) invertible in $M_{n}(A)$ and such that

(i) The *-algebra $\mathcal{A}$ generated by the entries $u_{i j}$ is everywhere dense in $A$;

(ii) The following identity holds:

$$
\phi\left(u_{i j}\right)=\sum_{k} u_{i k} \otimes u_{k j} .
$$

In this case we have the inclusion

$$
\phi(\mathcal{A}) \subseteq \mathcal{A} \otimes_{\text {alg }} \mathcal{A} .
$$

Let us stress that the above mentioned coassociativity and density properties are satisfied automatically.

Matrix groups generalize compact Lie groups (if $A$ is commutative the theory reduces to standard compact matrix groups).

The algebra $\mathcal{A}$ plays the role of the algebra of polynomial functions over $G$. The matrix $u \in M_{n}(A)$ correspond to the fundamental representation of the group $G$.

\section{Appendix B. Differential Calculus on quantum groups, Quantum Lie Algebras, Quantum universal envelopes}

B.1. Quantum Lie Algebras. There is a very important notion of a differential structure defined for quantum groups. The definitions of a quantum Lie algebra and of a quantum universal enveloping algebra depend on the introduced differential calculus. Therefore, we begin from giving the definition of the differential calculus.

First-order differential calculi are defined as certain bimodules $\Gamma$ over $\mathcal{A}$, equipped with a differential $d: \mathcal{A} \rightarrow \Gamma$. The space $\Gamma$ is a noncommutative counterpart of the usual module of 1-forms over a classical group, and $d$ generalizes the standard differential of functions.

It is important to mention that there is not a unique prescription to construct a differential calculus over a quantum group, and generally a given quantum group 
will possess a variety of non-equivalent calculi, each of them having a potential significance. It is surprising that the same situation appears in classical theory, where one can also use the methods of quantum groups to construct new differential calculi over the standard Lie groups. This opens interesting new possibilities in the study of classical Lie groups. In particular, it opens a possibility to extend the notion of dynamical symmetry, in the framework of classical groups.

In the quantum group theory a special role is played by so-called left-covariant and bicovariant differential calculi. In these cases [13] we can introduce the analogs of left and left/right actions of the group $G$ on $\Gamma$. If the module $\Gamma$ is left-covariant, then we can define its subspace $\Gamma_{\text {inv }}$, consisting of left-invariant '1-forms'. Quantum Lie algebra is then defined as the corresponding dual space, in other words $L=\Gamma_{\text {inv }}^{*}$.

If the calculus is bicovariant, then we can introduce a natural braid operator $\sigma: L \otimes L \rightarrow L \otimes L$, generalizing the classical transposition. Furthermore, in analogy with classical theory, we can define a quantum Lie bracket in the space $L$ generalizing the classical Lie bracket 13. The Lie bracket is defined as a linear operator $C: L \otimes L \rightarrow L$, and we can equivalently write $[x, y]=C(x \otimes y)$. This bracket satisfies the appropriate generalized Jacobi identity and braided-antisymmetricity conditions.

Following the classical theory, the quantum universal enveloping algebra for $(L,[]$,$) is defined as a unital associative algebra U(L)$ generated by relations

$$
x y-\sum_{i} y_{i} x_{i}=[x, y]
$$

where $x, y \in L$ and $\sum_{i} y_{i} \otimes x_{i}=\sigma(x \otimes y)$.

B.2. Representations of Quantum Groups and Quantum Lie Algebras. Having the Lie bracket and using (B.1) one can define representations of quantum Lie algebras and of the corresponding quantum universal enveloping algebras. It can be shown that every representation $v$ of $G$ in a finite-dimensional vector space $V$ naturally gives rise to a representation $S: U(L) \rightarrow \operatorname{End}(V)$ of the quantum universal enveloping algebra. Namely, let $v: V \rightarrow V \otimes \mathcal{A}$ be a (left) representation of the quantum group $G$ in a finite dimensional complex vector space $V$, in other words $v$ is linear, satisfies the condition

$$
(\mathrm{id} \otimes \phi) v=(v \otimes \mathrm{id}) v
$$

and $v$ is invertible, understood as an element of $\operatorname{End}(V) \otimes \mathcal{A}$. This corresponds to the classical requirements on representations of groups saying that the product of group elements is represented by composition of operators representing these elements, and the neutral element of a group is represented by the identity operator.

Every representation $v$ of $G$ in $V$ naturally generates a representation

$$
\delta=\delta_{v}: U(L) \rightarrow \operatorname{End}(V)
$$

of $U(L)$ in $V$ (if the differential calculus is bicovariant) or only of the Lie algebra $L, \delta: L \rightarrow \operatorname{End}(V)$ (if the differential calculus is left-covariant).

Moreover, if the differential calculus is *-covariant, which means that in the module $\Gamma$ of 1 -forms is defined the ${ }^{*}$-operation ${ }^{*}: \Gamma \rightarrow \Gamma$ induced by $*$ in $\mathcal{A}$, it makes sense to speak about hermiticity of the representation $\delta$. Namely, the ${ }^{*}$ operation on $\Gamma$ naturally induces the ${ }^{*}$-structure on the quantum Lie algebra $L$, via the formula $<f^{*}, \psi>=-<f, \psi^{*}>$ where $f \in L=\Gamma_{\text {inv }}^{*}$ and $\psi \in \Gamma_{\text {inv }}$. 
B.3. Quantum $S U(2)$ group. This quantum group is based on a $C^{*}$-algebra $A$ generated by elements $\left\{\alpha, \alpha^{*}, \gamma, \gamma^{*}\right\}$ satisfying the following relations:

$$
\begin{gathered}
\alpha \alpha^{*}+\mu^{2} \gamma^{*} \gamma=1 \quad \alpha^{*} \alpha+\gamma^{*} \gamma=1 \\
\gamma^{*} \gamma=\gamma \gamma^{*} \\
\alpha \gamma=\mu \gamma \alpha \quad \alpha \gamma^{*}=\mu \gamma^{*} \alpha,
\end{gathered}
$$

where $\mu \in[-1,1] \backslash\{0\}$. The comultiplication $\phi: A \rightarrow A \otimes A$ is given by

$$
\begin{aligned}
\phi(\alpha)=\alpha \otimes \alpha-\mu \gamma^{*} \otimes \gamma & \phi\left(\alpha^{*}\right)=\alpha^{*} \otimes \alpha^{*}-\mu \gamma \otimes \gamma^{*} \\
\phi(\gamma)=\gamma \otimes \alpha+\alpha^{*} \otimes \gamma & \phi\left(\gamma^{*}\right)=\gamma^{*} \otimes \alpha^{*}+\alpha \otimes \gamma^{*}
\end{aligned}
$$

The theory of representations of $S_{\mu} U(2)$ is very interesting from the point of view of our examples. This theory has many similarities to its classical counterpart-the theory of representations of $S U(2)$. Classical $S U(2)$ is obtained as a special case $\mu=1$.

The fundamental representation of $S_{\mu} U(2)$ is defined by the matrix

$$
u=\left(\begin{array}{cc}
\alpha & -\mu \gamma^{*} \\
\gamma & \alpha^{*}
\end{array}\right)
$$

It is easy to see that the defining relations for $S_{\mu} U(2)$ are equivalent to the unitarity property

$$
u^{*} u=u u^{*}=\left(\begin{array}{ll}
1 & 0 \\
0 & 1
\end{array}\right) .
$$

The fundamental representation enables us to build all other representations by using direct sums, tensor products and reduction procedures. Irreducible representations $u_{j}$ are numbered by half-integers $j$, and are $2 j+1$-dimensional. Every representation of an arbitrary compact quantum group can be decomposed into irreducible ones.

\section{REFERENCES}

[1] R P Feynman: Simulating Physics with Computers, Int Jour Theor Phys 21 (1982) 467-488

[2] P W Shor: Scheme for Reducing Decoherence in Quantum Computer Memory, Phys Rev A 52 (1995) R2493-R2496

[3] A M Steane: Error Correcting Codes in Quantum Theory, Phys Rev Lett 77 (1996) 793-797

[4] R Laflamme, C Miquel, J P Paz, W H Zurek: Perfect Error Correcting Code, Phys Rev Lett 77 (1996) 198-201

[5] Lu-Ming Duan. Guang-Can Guo: Quantum error avoiding codes versus quantum error correcting codes, quant-ph/9809057

[6] P Zanardi, M Rasetti: Noiseless Quantum Codes, Phys Rev Lett 79 (1997) 3306-3309

[7] P Zanardi, M Rasetti: Error Avoiding Quantum Codes, Mod Phys Lett B 25 (1997) 10851093

[8] D A Lidar. I L Chuang, K B Whaley: Decoherence Free Subspaces for Quantum Computation, quant-ph/9807004

[9] M Knill, R Laflamme: Theory of Quantum Error Correcting Codes, Phys Rev A55 (1997) 900-911

[10] A Perelomov: Generalized Coherent States and Their Applications, Texts and Monographs in Physics, Springer-Verlag (1986)

[11] M Chaichian, A Demichev: Introduction to Quantum Groups, World Scientific, (1996)

[12] S L Woronowicz: Differential Calculus on Compact Matrix Pseudogroups (Quantum Groups), Comm Math Phys 122 (1989), 125-170

[13] S L Woronowicz: Tannaka-Krein Duality for Compact Matrix Pseudogroups; Twisted SU(n) groups, Invent Math 93 (1988) 35-76 
[14] S Iwao: Knot and Conformal Field Theory Approach in Molecular and Nuclear Physics, Prog Theor Phys 83 (1990) 363-367

[15] A M Gavrilik: Quantum Groups in Hadron Phenomenology, hep-ph/9712411

[16] S L Woronowicz: Twisted SU(2) group; An example of a noncommutative differential calculus, RIMS Kyoto University 23 (1987) 117-181

[17] P Stachura: Bicovariant Differential Calculi on $S_{\mu} U(2)$, Lett Math Phys 25 (1992) 175-188

[18] S L Woronowicz: Compact Matrix Pseudogroups, Comm Math Phys 111 (1987) 613-665

[19] P Podleś: Quantum Spheres, Lett Math Phys 14 (1987) 193-202

[20] P Podleś: Differential Calculus on Quantum Spheres, Lett Math Phys 18 (1989) 107-119

[21] M Đurđevich: Geometry of Quantum Principal Bundles I, Comm Math Phys 175 (1996) $457-520$

[22] M Đurđevich: Geometry of Quantum Principal Bundles II, Rev Math Phys 9 (1997) 531-607

[23] M Đurđevich: Quantum Principal Bundles and Tannaka-Krein Duality, Rep Math Phys 38 (1996) 313-324

[24] M Đurđevich: Quantum Principal Bundles and Corresponding Gauge Theories, J Phys A30, (1997), 2027-2054

[25] M Đurđevich: Quantum Principal Bundles and Their Characteristic Classes, Quantum Groups and Quantum Spaces, Banach Center Publications, 40, Polish Scientific Publisher, Warsaw (1997)

[26] M Đurđevich: Quantum Classifying Spaces and Universal Quantum Characteristic Classes, Quantum Groups and Quantum Spaces, Banach Center Publications, 40, Polish Scientific Publisher, Warsaw (1997)

[27] H Makaruk: Fibre Bundles of Quantum Spheres, Proceedings of The 26-th Symposium on Mathematical Physics, Torun, December (1993)

[28] V G Drinfel'd: Quantum Groups, Proceedings of the International Congress of Mathematicians, Berkeley, (1986)

[29] L Fadeev, N Reshetikhin, L Takhtajan: Quantum Groups, Braid group, Knot Theory and Statistical Mechanics, World Scientific (1989) 87-110

[30] Lu-Ming Duan, Guang-Can Guo: Reducing Decoherence in Quantum Computer Memory with all Quantum Bits Coupling to the same Environment, Phys Rev A57 737-741

[31] L Viola, S Lloyd: Dynamical suppression of decoherence in two-state quantum systems, quant-ph/9803057

[32] L Viola, S Llovd: Decoherence control in quantum information processing: simple models, quant-ph/9809058

[33] L Viola, E Knill, S Lloyd: Dynamical decoupling of open quantum systems, quant-ph/9809071

[34] Lu-Ming Duan. Guang-Can Guo: Pulse Controlled Noise Suppressed Quantum Computation, quant-ph/9807072

[35] D Vitali, P Tombesi: Using parity kicks for decoherence control, quant-ph/9808055

[36] P Zanardi: Symmetrizing Evolutions, quant-ph/9809064 